Zbl Arbeitsmed 2021 · 71:109-117 https://doi.org/10.1007/s40664-020-00420-8 Eingegangen: 29. Juli 2020

Überarbeitet: 13. Dezember 2020

Angenommen: 16. Dezember 2020

Online publiziert: 29. Januar 2021

(c) Der/die Autor(en) 2021

\author{
Mike Schmidt ${ }^{1}$ Daniela Ohlendorf ${ }^{2}$ Rüdiger Reer ${ }^{1}$ David A. Groneberg ${ }^{2} \cdot$ \\ Eileen M. Wanke ${ }^{2}$ \\ 'Fakultät für Bewegungswissenschaft, Institut für Sport und Bewegungsmedizin, Universität Hamburg \\ Hamburg, Deutschland \\ ${ }^{2}$ Institut für Arbeits-, Sozial- und Umweltmedizin, Goethe-Universität, Frankfurt/Main, Deutschland
}

\title{
Berufsbezogene Zufriedenheit und Gesundheitswahrnehmung von Tanzpädagoginnen und -pädagogen
}

\section{Hintergrund}

Die sport- und bewegungsbezogene Vermittlung technischer, konditioneller, ästhetischer und kompositorischer Inhalte stellt die Hauptarbeit der tanzpädagogischen Tätigkeit dar [8, 16].

Der Aufrechterhaltung der Gesundheit von Tanzpädagog*innen (TP) kann eine Doppelfunktion zugeschrieben werden. Einerseits liegt sie in einem ganz persönlich-individuellen Interesse, um die eigene Lebensführung und existenzsichernde Berufsausübung $\mathrm{zu}$ gewährleisten. Andererseits kann ihr eine gesellschaftliche Funktion im Sinne einer erzieherischen, gesundheitsvermittelnden und verantwortungsübernehmenden Vorbildfunktion gegenüber den Schüler*innen zugeordnet werden [11, 19]. Umso erstaunlicher ist es, dass die gesundheitliche Situation solcher Lehrkräfte in der Vergangenheit selten erfasst wurde. Wanke et al. [16] berichteten, dass in ihrer Erhebung $77 \%$ der TP nie an einem Gesundheitsscreening teilgenommen haben. Neben der Erfassung unfallbedingter, akuter Verletzungen, die nach Erkenntnissen von Wanke et al. [17] in dieser Population als sehr gering eingeschätzt werden können, scheinen körperliche und mentale Belastungen eine größere Rolle zu spielen [21].

Als relativ gesichertes Wissen in der arbeitsmedizinischen Betrachtung gilt die Erkenntnis, dass eine allgemeine Berufszufriedenheit in direktem $\mathrm{Zu}$ - sammenhang mit verschiedenen berufsbezogenen Gesundheitsaspekten steht [4], ebenso wie der subjektiv bewertete Gesundheitszustand in Verbindung zum objektiven Gesundheitszustand gebracht werden kann [22]. Korrelationen zwischen Berufszufriedenheit, physischer und mentaler Gesundheit sowie Berufsstress und Fehlzeiten lassen sich ebenso für Lehrer*innen an Schulen zunehmend häufiger nachweisen [2]. Solche Beobachtungen konnten auch innerhalb der speziellen Untergruppe der Sportlehrer^innen festgestellt werden $[2,13]$. Eine vergleichbare Betrachtung anderweitig bewegungsvermittelnder Lehrkräfte wie den TP erscheint ebenso sinnvoll, um wertvolle Erkenntnisse über Berufszufriedenheit und den subjektiven Gesundheitszustand $\mathrm{zu}$ erhalten. Die Zahl der Berufstätigen in diesem Feld lassen sich allerdings nur schwierig erfassen. Nach Angaben des Deutschen Berufsverbandes für Tanzpädagogik e. V. (DBfT) ist von ca. $900 \mathrm{TP}$ auszugehen, die primär im künstlerischen Tanz tätig sind. Dies umfasst jedoch nur eine Teilgruppe organisierter TP, und es ist aufgrund der ungeschützten Berufsbezeichnung von einer deutlich größeren Population auszugehen. Erste Erkenntnisse aus dieser Berufsgruppe legen nahe, dass sowohl überaus positive als auch negative Aspekte vorliegen. So sehen sich nach Ergebnissen von Wanke et al. [18] 96,6\% der TP in ihrem Traumberuf. Andererseits sind für $85,5 \%$ der Befragten lange krankheitsbedingte Ausfälle nicht vertretbar, und $89,4 \%$ würden sogar unter Schmerzen weiterarbeiten [18]. Hier ist ein deutlicher sozioökonomischer Druck zu vermuten, der ebenfalls einen gesundheitsbelastenden Faktor darstellen könnte [8]. Zusätzlich sehen sich $78,7 \%$ der TP auch nach dem 64 . Lebensjahr noch in ihrem Beruf tätig [16]. Internationale Veröffentlichungen und dezidierte Untersuchungen $\mathrm{zu}$ diesen selbstwahrgenommenen Gesundheitsdimensionen dieser Population liegen derzeit kaum vor. Basierend auf der Nachfrage bewegungs- und tanzbezogener Angebote kann dieser Thematik jedoch eine nicht unerhebliche Relevanz zugeordnet werden [8]. Nach Angaben des Instituts für Demoskopie (IfD) Allensbach [5] tanzten zwischen 2016 und 2019 ca. 4,1 bzw. 4,3 Mio. Deutsche (ab 14 Jahren) in ihrer Freizeit häufig. Im Falle gelegentlichen Tanzens wird von einer etwa 6-mal so hohen Partizipation ausgegangen (zwischen 26,7 bzw. 27,5 Mio. Deutsche; [5]).

Das Ziel dieser explorativen, querschnittsbasierten Kohortenstudie war es, Daten über das eigene, berufsbedingte Gesundheitsempfinden, die Berufszufriedenheit sowie Belastungsfaktoren von TP im Rahmen einer anonymen Erhebung (online) zu sammeln. Darüber hinaus sollten mögliche geschlechtsspezifische Unterschiede und Zusammenhänge zwischen einzelnen Belastungsfaktoren herausgestellt wer- 


\begin{tabular}{|c|c|c|c|c|}
\hline Merkmal & $n$ & MW & SD & $95 \% \mathrm{KI}$ \\
\hline \multicolumn{5}{|c|}{ Alter (Jahre) } \\
\hline Gesamt & 232 & 43,1 & 11,0 & $41,7-44,5$ \\
\hline Frauen & 181 & 42,0 & 10,5 & $40,5-43,5$ \\
\hline Männer & 51 & 46,9 & 11,9 & $43,6-50,3$ \\
\hline \multicolumn{5}{|c|}{ Größe $(\mathrm{cm})$} \\
\hline Gesamt & 232 & 170,0 & 7,7 & $169,0-171,0$ \\
\hline Frauen & 181 & 167,8 & 6,2 & $166,9-168,7$ \\
\hline Männer & 51 & 177,8 & 7,6 & $175,7-179,9$ \\
\hline \multicolumn{5}{|c|}{ Gewicht (kg) } \\
\hline Gesamt & 232 & 61,7 & 10,8 & $60,3-63,1$ \\
\hline Frauen & 181 & 58,3 & 8,2 & $57,1-59,5$ \\
\hline Männer & 51 & 74,0 & 10,1 & $71,1-76,8$ \\
\hline \multicolumn{5}{|c|}{$B M I\left(k g^{*} m^{-2}\right)$} \\
\hline Gesamt & 232 & 21,3 & 2,7 & $20,9-21,6$ \\
\hline Frauen & 181 & 20,7 & 2,4 & $20,3-21,0$ \\
\hline Männer & 51 & 23,4 & 2,6 & $22,6-24,1$ \\
\hline
\end{tabular}

den. Eine Beeinflussung der Ergebnisse durch die COVID-19-Pandemie konnte ausgeschlossen werden, da die Erhebung vor Beginn der Pandemie lag.

\section{Methodik}

\section{Studiendesign und Studien- population}

Im Rahmen einer retrospektiven Querschnitts-Kohorten-Studie wurden TP mit einem Online-Fragebogen (in englischer und deutscher Sprache über „survey monkey“) befragt.

Die folgenden Einschlusskriterien wurden vor der Befragung festgelegt:

- Hauptberufliche Ausübung der Tätigkeit als Tanzpädagog*in

- Volljährigkeit ( $\geq 18$ Jahre)

- Angestellt oder freiberuflich

- Überwiegende Arbeit in mindestens einem der folgenden künstlerischen Tanzstile: klassischer Tanz (Ballett), Modern/Jazz Dance oder zeitgenössischer Tanz

- Wohnhaft in Deutschland

Die Studie befolgt wissenschaftsethische Kriterien. Eine Zustimmung zur ethischen Unbedenklichkeit der Studienprozedur erfolgte seitens der Ethikkommission der Charité - Universitätsmedizin Berlin.

\section{Studiendurchführung}

Im Rahmen einer Prätestung an einer kleineren Stichprobe von Sportlehrkräften wurden grundlegende Probleme im Umgang mit dem Erhebungsinstrument ausgeschlossen. Die Haupterhebung erfolgte über einen Zeitraum von 3 Monaten. Der Zugang zur anonymen Befragung wurde auf digitalem Wege mit Unterstützung des Deutschen Berufsverbands für Tanzpädagogik e. V. (DBfT e.V.), der Royal Academy of Dance (Germany), der Stiftung TANZ und dem Gemeinnützigen Verein für Tanzmedizin (ta.med e. V.) an potenzielle Kandidaten verschickt.

Um die Rücklaufquote zu erhöhen, wurde mit 3 Erinnerungsmails im gesamten Zeitraum auf die Befragung hingewiesen sowie die Bitte zur Weiterleitung an Kollegen geäußert. Eine finale Rücklaufquote ließsich aufgrund fehlender Informationen zur Gesamtpopulation der TP in Deutschland nicht berechnen.

\section{Datenanalyse}

\section{Fragebogeninhalte}

Im Rahmen eines Komplex-Fragebogens wurden u.a. anthropometrische (Alter, Größe, Gewicht), soziodemographische (Bildungs- sowie Berufsabschluss, monatliches Einkommen) sowie in Anlehnung an Wanke et al. [16] tanzspezifische und berufsbezogene (Tanzstile, Berufserfahrung) Merkmale erfragt. Darüber hinaus wurden unter Berücksichtigung und Modifizierung übergeordneter Dimensionen (z. B. ,work-privacy conflict“) des COPSOQ [9] psychosoziale Berufsfaktoren, die Berufszufriedenheit sowie der subjektive Gesundheitszustand exploriert. Der subjektive Gesundheitszustand wurde auf einer 6-stufigen Skala $(1=$ „sehr gut“ bis $6=$,ungenügend“ $)$ eruiert. Zur Einschätzung der allgemeinen Berufszufriedenheit sowie dem Zustimmungsgrad weiterer psychosozialer Berufsfaktoren wurden anstelle 5-stufiger [9] 7-stufige Likert-Skalen („Die Antwort stimmt...": „,voll und ganz“, „trifft eher zu“, „,etwas“, „teils/teils“, „weniger“, „eher nicht“, „gar nicht“) verwendet.
Die Datenverarbeitung erfolgte mit Microsoft Excel 2010 (Microsoft Excel (2010), Microsoft Corporation, Redmond, WA, USA). Die deskriptive und analytische Statistik wurde mit IBM SPSS 25 (IBM SPSS Statistics für Windows, Version 25.0, IBM Corp, Armonk, NY, USA) durchgeführt. Allen Tests lag eine zweiseitige Testung und ein Signifikanzniveau von $\alpha=0,05$ zugrunde. Die Gruppenunterschiede innerhalb der ordinalskalierten Fragebogenitems, wie den Likert-Skalen, wurden mittels Mann-Whitney-U-Test überprüft. Neben der Angabe der Irrtumswahrscheinlichkeit $p$ wurde als Effektstärke $\mathrm{r}_{\mathrm{ES}}$ mit $\mathrm{r}_{\mathrm{ES}}=\mathrm{z} / \sqrt{ } n$ angegeben. Zur Identifizierung möglicher Zusammenhänge zwischen den einzelnen Fragebogenitems wurden Spearman-KorrelationsKoeffizienten $\left(r_{\mathrm{sp}}\right)$ berechnet. 
Zbl Arbeitsmed 2021 · 71:109-117 https://doi.org/10.1007/s40664-020-00420-8

(c) Der/die Autor(en) 2021

M. Schmidt · D. Ohlendorf · R. Reer · D. A. Groneberg · E. M. Wanke

\section{Berufsbezogene Zufriedenheit und Gesundheitswahrnehmung von Tanzpädagoginnen und -pädagogen}

\section{Zusammenfassung}

Hintergrund. Der eigene Körper ist das zentrale Arbeitsinstrument eines*einer Tanzpädagog*in (TP) innerhalb der Bewegungsvermittlung. Bisher fehlen Erkenntnisse über die subjektive Wahrnehmung der eigenen berufsassoziierten Gesundheit und Zufriedenheit sowie die Identifizierung gesundheitsbelastender Berufsmerkmale. Methodik. Im Rahmen einer fragebogenbasierten Querschnittserhebung wurde eine Kohorte von TP in Deutschland zur eigenen Gesundheit und generellen Berufszufriedenheit und belastenden Aspekten im Zusammenhang mit ihrer Berufsausübung untersucht. Zusätzlich wurden allgemeine anthropometrische und soziodemographische Merkmale erfasst. Neben der Betrachtung der Gesamtkohorte wurde auf geschlechtsspezifi- sche Unterschiede getestet. In die statistische Analyse wurden $n=232$ TP (m: 51/w: 181) im Alter von 43,1 $\pm 11,0$ Jahren eingeschlossen. Ergebnisse. Der allgemeine Gesundheitszustand wurde von $85,3 \%$ der Befragten mit "befriedigend" (26,1\%) bis "sehr gut" $(14,7 \%)$ beurteilt. 59,2\% der Tanzpädagog*innen schätzten ihre Gesundheit "gut" (35,3\%) bis „sehr gut" ein. Es herrschte eine hohe Zufriedenheit mit der eigenen Berufsausübung für $80 \%$ der Teilnehmenden. Die TP fühlten sich überwiegend in der Lage (trifft "voll \& ganz" bzw. „eher zu“"), mit den physischen (75,7\%) und psychischen Berufsanforderungen $(70,3 \%)$ umzugehen. Als belastende Berufsmerkmale in der Eigenwahrnehmung können neben Zukunftsängsten $(51,5 \%)$ vor allem arbeitsorganisatorische (fehlende Zeit für Familie und Freunde bei 28,4\%) und ökonomische Aspekte (Einkommensunsicherheit bei $61,0 \%$ und fehlende Altersabsicherung bei $65,7 \%$ ) herausgestellt werden. Diskussion. Die Berufsausübung als TP geht mit einer hohen generellen Zufriedenheit und einem positiven Empfinden des eigenen Gesundheitszustandes einher. Eine Bestätigung dieser positiven Ergebnisse durch Verletzungs- und Erkrankungsstatistiken steht noch aus. Darüber hinaus wäre eine Verbesserung arbeitsorganisatorischer und ökonomischer Aspekte wünschenswert.

Schlüsselwörter

Berufszufriedenheit · Berufliche Gesundheit . Subjektiver Gesundheitszustand · Tanz . Pädagoge/in

\section{Job-related satisfaction and health perception of dance teachers}

\section{Abstract}

Background. The dance teacher's own body is considered to be the central working instrument within the movement mediation. Up to now there is a lack of knowledge about the subjective perception of one's own occupationally associated health and satisfaction as well as the identification of occupational characteristics that are detrimental to health.

Material and methods. Within the framework of a questionnaire-based cross-sectional survey, a cohort of dance teachers in Germany were investigated about dimensions of their own health and general job satisfaction in connection with their profession as a dance teacher. Likert scales were primarily used for these assessments. Additionally, general anthropometric and sociodemographic cha- racteristics were recorded. Beside examining the overall cohort, gender-specific differences were tested. The statistical analysis included $n=232$ dance teachers (male 51/female 181) aged $43.1 \pm 11.0$ years.

Results. The general state of health was rated as satisfactory (26.1\%) to very good $(14.7 \%)$ by $85.3 \%$ of those surveyed. Of the dance teachers $59.2 \%$ even rated their health as good (35.3\%) to very good. There was a high degree of satisfaction with their own professional practice for $80 \%$ of the participants. Most of the dance teachers felt that they were able to deal with the physical (75.7\%) and psychological demands of their profession (70.3\%). In addition to fears about the future $(51.5 \%)$, the main burdensome professional characteristics on dance teachers' self-perception were organizational aspects of work (lack of time for family and friends in 28.4\%) and economic aspects (income insecurity in $61.0 \%$ and lack of old age security in 65.7\%).

Conclusion. The profession as a dance teacher is accompanied by a high level of general satisfaction and a positive perception of one's own state of health. A confirmation of these positive results by injury and illness statistics is still pending. In addition, an improvement in work organization and economic aspects would be desirable.

\section{Keywords}

Job satisfaction - Occupational health . Subjective health status - Dance - Pedagogue

\section{Ergebnisse}

\section{Anthropometrische und soziodemographische Merkmale der Stichprobe}

Im gesamten Erhebungszeitraum haben $n=241$ TP erfolgreich die Umfrage abgeschlossen. Für die finale Datenanalyse wurden die Befragungen von $n=232$ TP betrachtet, bei denen keine fehlen- den Werte für die allgemeinen Personenmerkmale Geschlecht, Alter, Größe und Gewicht zu verzeichnen waren.

- Tab. 1 gibt eine Übersicht über wichtige anthropometrische Merkmale der Stichprobe. Mit einem Anteil von $78 \%$ ( $n=181)$ überwogen die weiblichen TP deutlich ihre männlichen Kollegen (22\% bzw. $n=51$ ).

Die TP waren zumeist selbstständig tätig (72,8\%; • Abb. 1) und der Groß- teil $(90,1 \%)$ besaß ein monatliches Nettoeinkommen $\leq 3500 €$. Etwa drei Viertel der Rückmeldungen (78,7\%) lag bei weniger als $2500 €$ und etwa ein Drittel $(31,2 \%)$ bei weniger als $1000 €$ pro Monat. Es bestand ein signifikanter Unterschied im monatlichen Verdienst zwischen weiblichen und männlichen Lehrkräften $\left(r_{E S}=0,22 ; p=0,008\right)$. Lediglich $24,7 \%$ der Lehrkräfte gaben an, über keine Hochschulreife zu verfügen und 8,5\% 


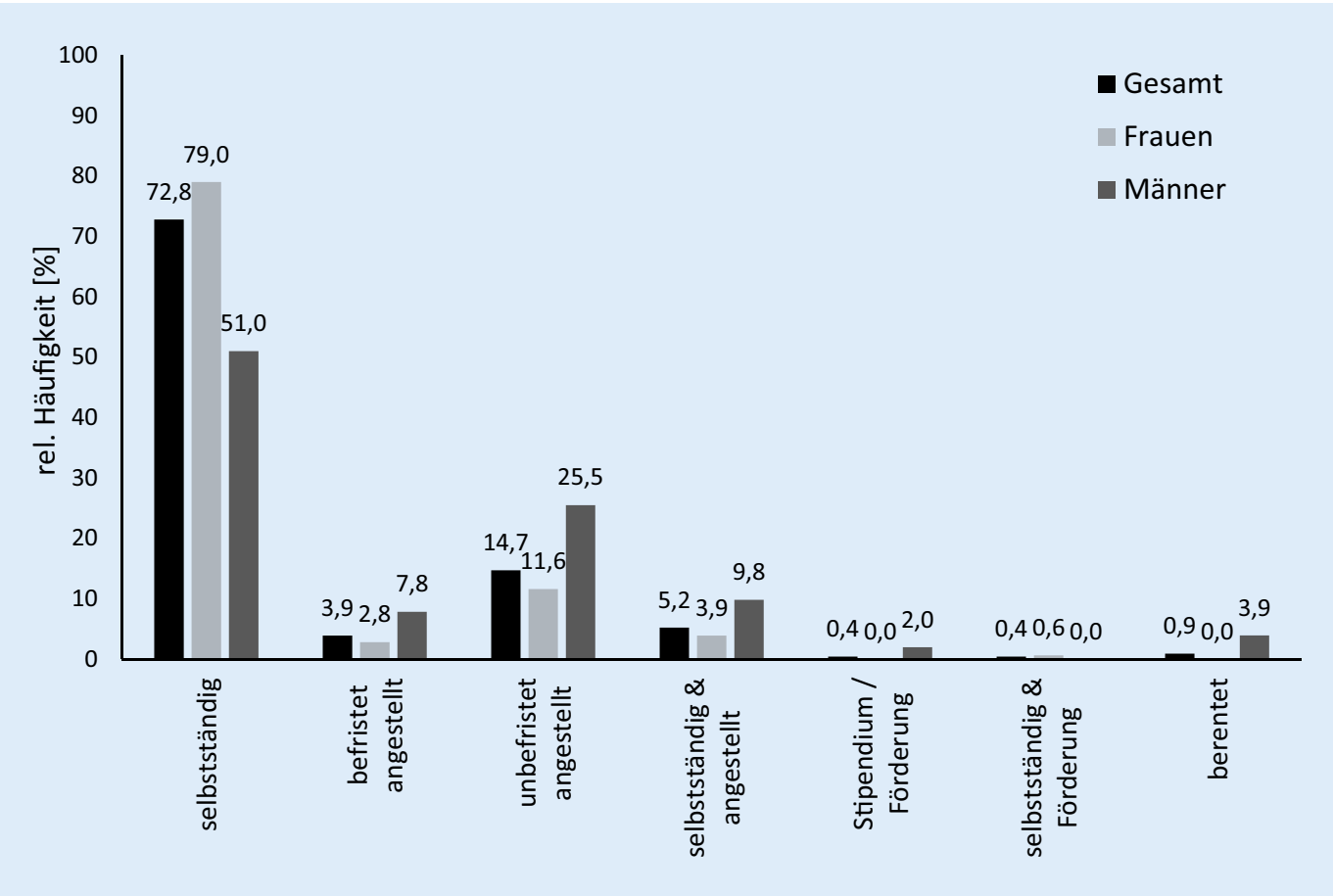

Abb. $1 \triangleleft$ Anstellungsverhältnisse von Tanzpädagog*innen $(n=232)$

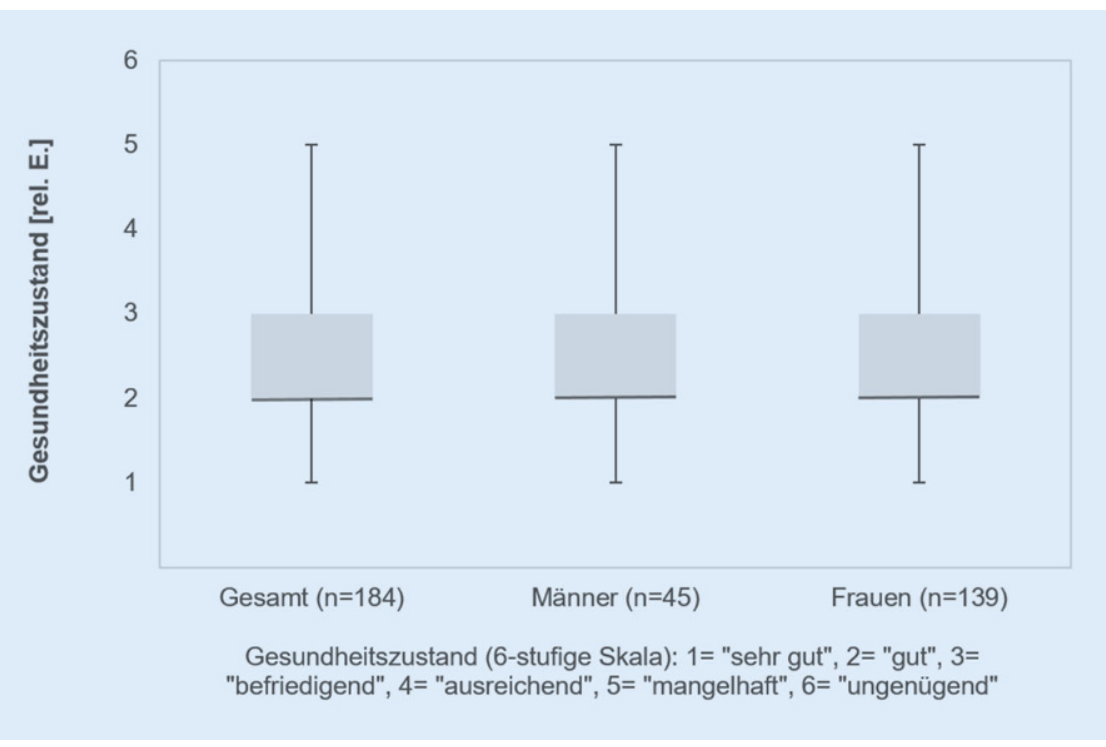

Abb. $2 \Delta$ Allgemeiner Gesundheitszustand von Tanzpädagog*innen

absolvierten sogar ein Hochschulstudium.

\section{Subjektive Beurteilung des allgemeinen Gesundheits- zustandes}

Der eigene Gesundheitszustand wurde von $85,3 \%$ mit „befriedigend“ (26,1\%), "gut“ (44,5\%) oder „sehr gut“ (14,7\%) bewertet. Lediglich $14,7 \%$ beurteilten ihren Gesundheitszustand mit ,ausrei- chend“ $(8,2 \%)$ oder gar „mangelhaft“ (6,5\%). Die Einschätzung „ungenügend“ wurde von niemandem ausgewählt. Die geschlechtsspezifischen Unterschiede waren nicht signifikant (• Abb. 2).

\section{Allgemeine Berufszufriedenheit und -umstände}

Es konnte eine insgesamt sehr hohe allgemeine Zufriedenheit mit der Berufsausübung als TP gezeigt werden.
$34,4 \%$ der TP waren „voll und ganz“ zufrieden mit ihrem Beruf, gefolgt von $45,6 \%$, die die zweithöchste Zufriedenheitseinschätzung „trifft eher $z u^{\text {“ }}$ angaben. Lediglich 12,6\% der Befragten beurteilten ihren Zufriedenheitsgrad mit „teils/teils“ oder schlechter.

Ähnliches ließ sich für den Kontakt zu den Kollegen abbilden. Mit „voll und ganz" bzw. „trifft eher zu“ stimmten $45,0 \%$ bzw. 40,3\% der TP einem angenehmen Kollegenkontakt zu. Darüber hinaus sahen sich die Lehrkräfte in der Lage, den täglichen, körperlichen Belastungen gerecht zu werden. Etwas geringer fiel die Zustimmung zum Umgang mit den psychischen Berufsanforderungen sowie Stress- und Konfliktsituationen aus. Die größte Aussageheterogenität konnte für die Frage nach ausreichend Zeit und Energie für Familie und Freunde verzeichnet werden. Diese wurde von $28,4 \%$ tendenziell negiert („weniger“ bis "gar nicht“) bzw. von $51,7 \%$ aller mit „teils/teils“ oder schlechter bewertet (• Abb. 3).

Es zeigten sich geschlechtsspezifische Unterschiede im Umgang mit Stress- und Konfliktsituationen $\left(\mathrm{r}_{\mathrm{ES}}=0,19 ; p=0,003\right)$ sowie dem Empfinden den psychischen Berufsanforderungen gerecht $\mathrm{zu}$ werden $\left(r_{\mathrm{ES}}=0,14 ; p=0,039\right)$. Die weiblichen TP 


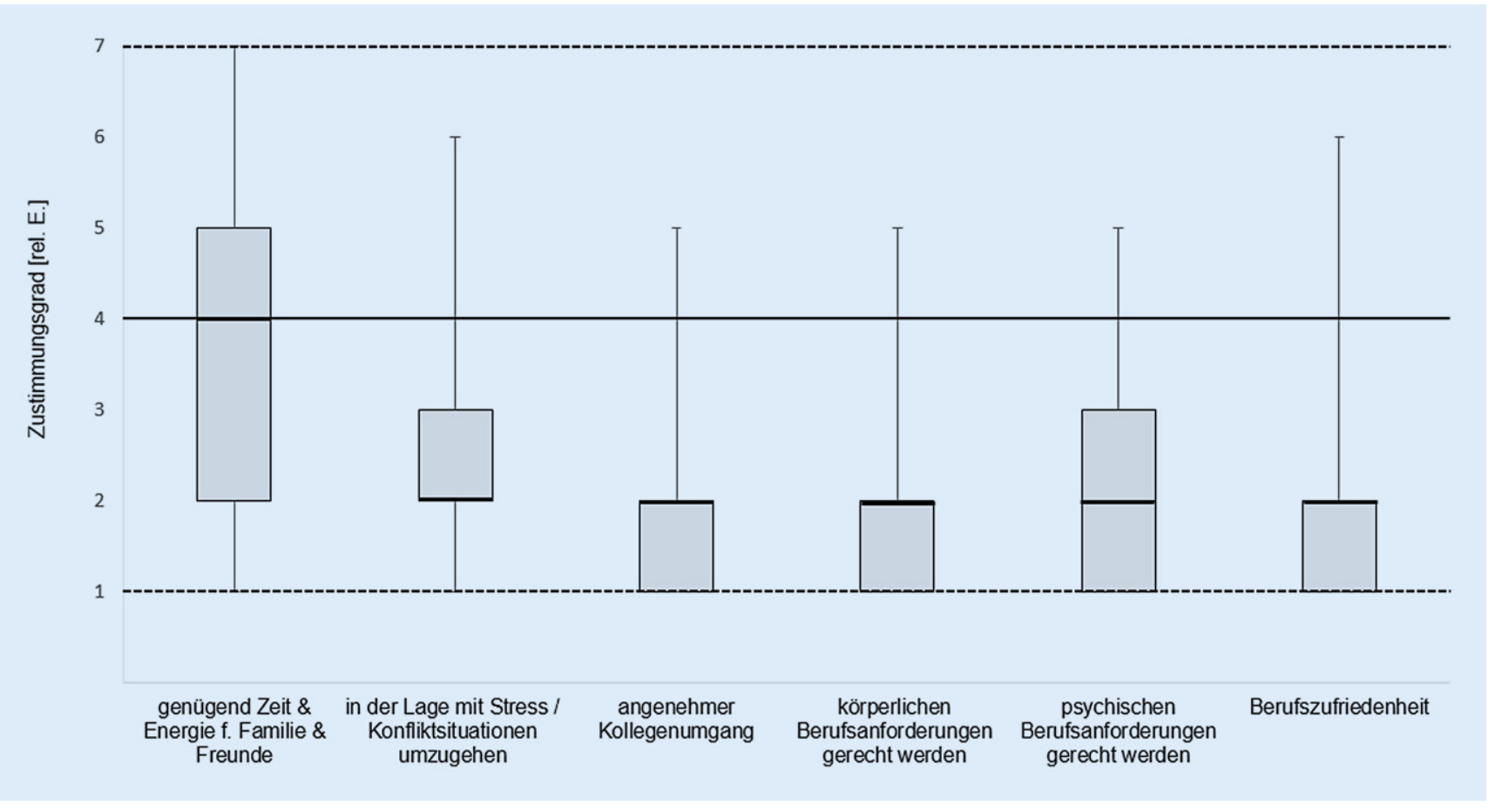

Abb. 3 A BelastungsbeurteilungallgemeinerBerufsumstände.Zustimmungsgrad (7-stufige Likert-Skala): $1=$ " voll und ganz",

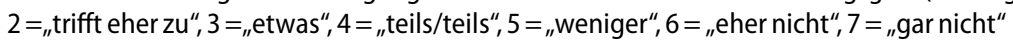

sahen sich in beiden Merkmalen etwas stärker belastet als ihre Kollegen.

\section{Beurteilung weiterer Berufs- aspekte als Belastungsfaktoren}

- Abb. 4 gibt einen Überblick über Berufsaspekte und das mit diesen jeweils assoziierte Belastungsempfinden der TP. Es wurde deutlich, dass mehr als die Hälfte $(51,5 \%)$ Zukunftsängste äußerten und $61,0 \%$ der Befragten einen belastenden Faktor in der Absicherung ihres Einkommens sahen. Weiterhin belastete $65,7 \%$ aller TP der Aspekt der Altersabsicherung. Hier zeigte sich ein signifikanter geschlechtsspezifischer Unterschied. So ging von männlichen TP ein höherer Besorgnisgrad aus als von den weiblichen $\mathrm{TP}\left(\mathrm{r}_{\mathrm{ES}}=0,17 ; p=0,012\right)$.

Die Fragen nach einem vorzeitigen Karriereende, Unsicherheiten in einem konkreten Engagement, möglichem Konkurrenzdruck sowie der körperlichen Belastung im Berufszusammenhang wurden mit geringerer Besorgnis verzeichnet. In diesen Merkmalen konnten keine geschlechtsspezifischen Unterschiede registriert werden.

\section{Zusammenhänge zwischen Gesundheitszustand, Zufriedenheit und Einkommen}

Es konnte festgestellt werden, dass zwischen der Bewertung des eigenen Gesundheitszustandes und den dargestellten Berufsmerkmalen (- Abb. 3 und 4) lediglich kleine oder keine Zusammenhänge (alle Korrelationskoeffizienten $<0,5)$ gefunden werden konnten. Der höchste Zusammenhang zum Gesundheitszustand zeigte sich in Verbindung mit der Beurteilung der körperlichen Belastung $\left(\mathrm{r}_{S \mathrm{P}}=-0,43 ; p<0,000\right)$. Die Korrelationen des Gesundheitszustandes zur generellen Berufszufriedenheit $\left(\mathrm{r}_{\mathrm{SP}}=0,18 ; p=0,019\right)$ und dem Einkommen $\left(\mathrm{r}_{\mathrm{SP}}=-0,19 ; p=0,029\right)$ fielen äußerst klein aus. In direkterer Beziehung standen die Berufszufriedenheit und das Einkommen, jedoch ebenfalls nur mit einem kleinen linearen Zusammenhang $\left(\mathrm{r}_{\mathrm{SP}}=-0,37 ; p<0,000\right)$.

\section{Berufsmerkmale in der Belastungsbeurteilung mit direktem Zusammenhang}

- Tab. 2 gibt eine Übersicht über alle mittleren und hohen Zusammenhänge zwischen den einzelnen Berufsmerkmalen, die in den $\bullet \mathbf{A b b} .3$ und 4dargestellt sind. Insgesamt konnten zwei hohe und acht mittlere positive Zusammenhänge ermittelt werden. Die beiden hohen Korrelationen bestanden jeweils zwischen dem Merkmal „Einkommenssicherheit" und den Merkmalen "Zukunftsangst“ $\left(\mathrm{r}_{\mathrm{SP}}=0,803 ; p<0,000\right)$ bzw. „Altersabsicherung" $\left(\mathrm{r}_{\mathrm{SP}}=0,741 ; p<0,000\right)$. Daraus kann geschlossen werden, dass Personen, die sich von ihrer Einkommenssituation belastet fühlten, ebenfalls in ihrer Altersabsicherung sowie Zukunftssicherung Besorgnis erregende Berufsaspekte sahen.

Merkmale zur Einschätzung der physischen und psychischen Anforderungen in Verbindung zu Merkmalen mit berufswirtschaftlichem und -organisatorischem Hintergrund zeigten maximal kleine Zusammenhänge. Hierbei wurde der größte Zusammenhang $\left(\mathrm{r}_{\mathrm{SP}}=0,470\right.$; $p<0,000)$ zwischen der körperlichen Be- 


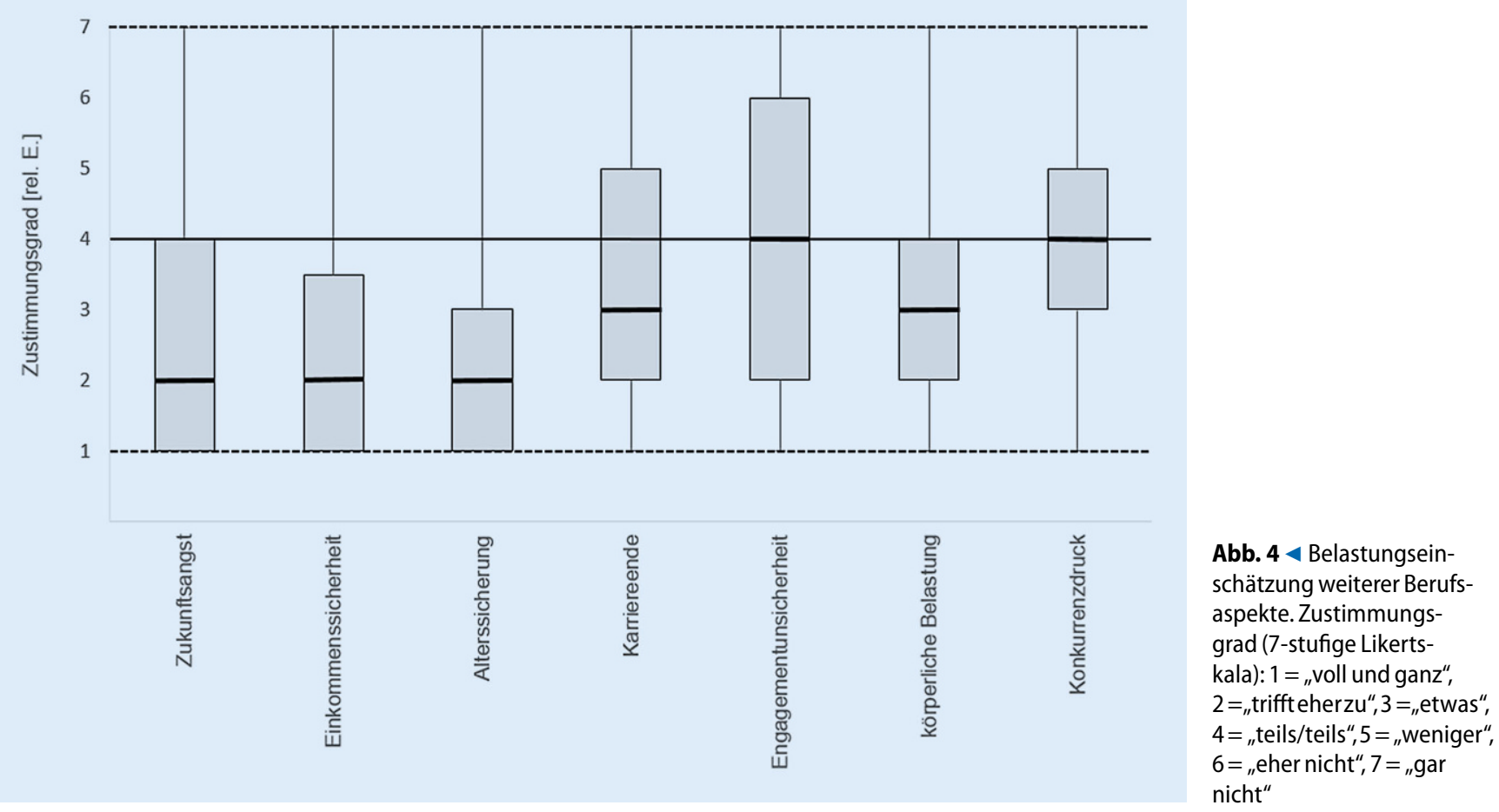

lastung und der Angst vor einem vorzeitigen Karriereende konstatiert. Weiterhin bestand zwischen der Beurteilung physischer Anforderungen und den körperlichen Belastungen nur ein sehr geringer Zusammenhang $\left(\mathrm{r}_{\mathrm{SP}}=-0,292 ; p<0,000\right)$.

\section{Diskussion}

\section{Subjektiver Gesundheitszustand}

Der subjektiv wahrgenommene Gesundheitszustand kann für die gesamte Stichprobe als gut beurteilt werden, wobei keine geschlechtsbezogenen Unterschiede bestehen. Der Personenanteil (59,2\%) mit einer guten oder sehr guten Beurteilung entspricht etwa den Beobachtungen in der deutschen Gesamtbevölkerung innerhalb gleicher Altersstrukturen [10]. So bewerten ca. $55 \%$ der $40-$ bis 49-jährigen Männer und Frauen ihren subjektiven Gesundheitszustand mit gut bis sehr gut [10]. Es kann vermutet werden, dass für diesen Großteil ebenfalls ein guter objektiver Gesundheitszustand vorliegt [22], auch wenn festzuhalten ist, dass selbstbewertete und objektive Gesundheit nicht gleichzusetzen sind $[10,22]$, sondern vielmehr als Indikator zu betrachten wären. Interessant ist, dass laut Robert Koch-Institut [10] in der deutschen Referenzpopulation lediglich etwa $2 \%$ eine schlechte Bewertung ihrer Gesundheit vorgenommen haben. Der Anteil innerhalb der TP mit 6,5\% (Bewertung: „mangelhaft“) fällt deutlich höher $(\approx 3$-fach) aus. Es ist zu erwarten, dass für diese Untergruppe auch objektive Gesundheitseinschätzungen (z. B. Krankheitsprävalenzen) schlechter ausfallen [22]. Aus den Beobachtungen muss vermutet werden, dass positive Gesundheitseffekte durch die Berufsausübung gering ausfallen und in gleichem $\mathrm{Ma}$ ße nicht von bedeutenden Verzerrungen im Kontext von Healthier-Worker-Effekten [6] auszugehen ist, bedürfen jedoch weiterer objektiver Gesundheitseinschätzungen.

\section{Generelle Berufszufriedenheit}

Bezogen auf die generelle Berufszufriedenheit konnte ein sehr hoher $\mathrm{Zu}$ friedenheitsgrad eruiert werden. Dies untermauert die Feststellung von Wanke et al. [18], dass es sich für die große Mehrheit um die Ausübung des Traumberufes handelt und stellt zusätzlich einen günstigen Umstand für eine gesundheitserhaltende Berufsausübung dar. Eine mögliche Erklärung für dieses Phänomen könnte sein, dass sich ein Großteil der Population in einer selbstständigen Arbeitssituation wiederfindet und somit über die direkten Arbeitsinhalte und die einhergehenden arbeitsstrukturellen Bedingungen entscheiden kann. So zeigten Skaalvik und Skaalvik [14] an 2569 Lehrer*innen den direkten Zusammenhang zwischen Arbeitszufriedenheit und arbeitsbezogener Autonomie $(r=0,37)$. Im Umkehrschluss kann ebenso vermutet werden, dass durch den hohen Zufriedenheitsgrad ein sehr geringes Risiko für ein berufsbedingtes Burnout besteht.

\section{Berufsbelastung}

TP sehen sich in der Lage, mit bestehenden körperlichen und psychischen Anforderungen umzugehen und diesen gerecht zu werden. Das kollegiale Berufsumfeld wird als angenehm empfunden. Es lässt sich vermuten, dass TP in einer Vielzahl ihrer direkten Berufsaufgaben, wie der sportmotorischen, choreographischen und ästhetischen Vermittlung, ein hohes Gefühl von Kontrolle und Kohärenz zu den eigenen Berufsanforderungen sehen und diese Bestandteile als gesundheitsstärkende Ressource wirken könnten. Die geschlechtsspezifischen Unterschiede zu Gunsten der männlichen TP mit einem geringeren Stress- 
Tab. 2 Zusammenhänge zwischen Belastungseinschätzungen einzelner Berufsmerkmale (dargestellt sind alle mittleren und hohen Spearman-Korrelationskoeffizienten [rsp] zwischen einzelnen Fragebogenitems zur Einschätzung berufsassoziierter Belastungen), $n=232$

\section{Merkmalspaarung}

Einkommenssicherheit

Zukunftsangst

Psychische Anforderungen

Altersabsicherung

Zukunftsangst

Einkommenssicherheit

Vorzeitiges Karriereende

Psychische Anforderungen

Zukunftsangst
Einkommenssicherheit

Zukunftsangst
Altersabsicherung
Altersabsicherung
Physische Anforderungen
Vorzeitiges Karriereende
Unsicherheit in Engagement
Unsicherheit in Engagement
Unsicherheit in Engagement
Umgang mit Stress- \&
Konfliktsituationen

Vorzeitiges Karriereende

\begin{tabular}{ll} 
rSP & $\boldsymbol{p}$ \\
\hline 0,803 & $<0,000$ \\
0,741 & $<0,000$ \\
0,665 & $<0,000$ \\
0,563 & $<0,000$ \\
\hline 0,549 & $<0,000$ \\
\hline 0,539 & $<0,000$ \\
0,534 & $<0,000$ \\
0,525 & $<0,000$ \\
\hline 0,508 & $<0,000$ \\
\hline 0,507 & $<0,000$
\end{tabular}

und Konfliktempfinden sowie einem höheren Empfinden, psychischen Berufsanforderungen gerecht $\mathrm{zu}$ werden, lieBen sich zeigen. Diese allgemeine Tendenz zeichnet sich auch in der Literatur ab $[1,6]$. Von einer praktischen Relevanz kann jedoch aufgrund der kleinen Effekte nicht ausgegangen werden. Zusätzlich können diese Geschlechtsunterschiede nach Alters- oder Bildungsgradadjustierung unter Umständen verschwinden [7]. Weiterhin zeigen die Ergebnisse, dass die Berufsausübung mit zeitlichen und energetischen Engpässen für das soziale Umfeld (Familie/Freunde) verbunden sein kann. Es lässt sich jedoch keine einheitliche Situation innerhalb der Stichprobe feststellen. Auf welche Ursache dieser Umstand zurückzuführen ist, bleibt spekulativ. Auffällig ist, dass TP vor allem besorgt sind über ihre Zukunfts-, Einkommens- sowie Alterssicherung. Diese Merkmale belasten die TP deutlich. Schaut man sich den hohen Anteil selbstständig Berufstätiger und das zumeist niedrige Einkommen an, erscheint dies nachvollziehbar und deckt sich mit früheren Erkenntnissen [18]. Zusätzlich ist zu vermuten, dass die COVID-19-bedingten Einschränkungen $\mathrm{zu}$ einer Verschlechterung der Berufssituation und sozioökonomischen Lage führen könnten.

\section{Körperliche Arbeit mit ambivalenter Wirkung}

Von der eigenen Körperarbeit scheinen sowohl positive als auch belastende Wir- kungen auszugehen. So sehen sich die TP einerseits in der Lage, den physischen Anforderungen gerecht zu werden, andererseits wird die körperliche Arbeit als Belastungsfaktor wahrgenommen. Diese ambivalente Wirkung der physischen Aktivität im Beruf als TP lässt sich ebenfalls aus den Arbeiten von Dahlström [3], Schmidt et al. [12] und Wanke et al. [20] ableiten. So belegen Dahlström [3] und Wanke et al. [20], dass körperliche Beanspruchungen des Herz-Kreislauf-Systems im Unterrichtsgeschehen in ihren Höchstbelastungen durchaus submaximale bis maximale Intensitäten erreichen können. Andererseits fallen durchschnittliche HerzKreislauf-Belastungen im Unterrichtsverlauf häufig gering bis moderat aus [20], und mit der Berufsausübung kann nicht automatisch eine höhere Fitness verbunden werden [12]. Eine ähnliche Beobachtung im Hinblick auf die Wirkung berufsbezogener körperlicher Aktivität lässt sich in der Studie von Bogaert et al. [2] für Sportlehrer*innen finden. Einerseits zeigten sie im Vergleich $\mathrm{zu}$ ihren restlichen Lehrerkolleg*innen eine signifikant bessere mentale Gesundheit sowie geringere Stresslevel und eine Begründung über eine höhere physische Aktivität im Zuge ihrer Berufsausübung erscheint naheliegend. Demgegenüber stellen die Autoren jedoch fest, dass in der Gesamtstichprobe aller Lehrkräfte ein höherer Umfang physischer Berufsaktivität teilweise mit geringeren Gesundheitsoutcomes assoziiert war [2]. Sie erklären ihre Ergebnisse mit dem spezifischen Aufgabencharakter der im Rahmen der Lehrtätigkeit ausgeführten physischen Aktivitäten [2]. Solche Unterschiede in der Arbeit mit dem eigenen Körper und eine hohe Individualität im Unterrichtsprozess lassen sich ebenso für die TP vermuten. So bringt beispielsweise der Einfluss der $\mathrm{zu}$ unterrichtenden Zielgruppe einen deutlichen Belastungsunterschied mit sich [21]. Festzuhalten ist, dass die größte Assoziation mit einem vorzeitigen Karriereaus zur körperlichen Belastung bestand und somit dem Umgang und der differenzierten Betrachtung dieser berufsbezogenen Körperarbeit eine Schlüsselrolle zugeordnet werden muss.

\section{Limitationen}

Da es sich um eine Fragebogenerhebung handelte, konnte nicht ausgeschlossen werden, dass die abgebildeten Belastungseinschätzungen die tatsächlichen Zustände der untersuchten Population leicht über- oder unterschätzen. Teilweise verweigerte Antworten könnten $\mathrm{zu}$ solchen Verzerrungen führen. So haben beispielsweise 20,7\% der Stichprobe keine Bewertung zum eigenen allgemeinen Gesundheitszustand abgegeben. Ob dadurch eine Überschätzung und damit positivere Sicht auf die Stichprobe zustande kam, kann letztlich nicht beantwortet werden. Ähnliches gilt für Fragebogenitems zu sensiblen Personenmerkmalen wie beispielsweise dem monatlichen Verdienst. Trotz Anonymität wurde dieses Item von $41,5 \%$ nicht beantwortet. Von einer bewussten Verfälschung bestehender Antworten gehen die Autoren jedoch aufgrund des anonymen und freiwilligen Befragungscharakters nicht aus. Zudem erfolgte die Akquise vordergründig über Verbandsstrukturen, mit denen von einer gewissen Vertraulichkeit auszugehen war. Die bereits in früheren Arbeiten $[8,21]$ und erneut beschriebenen Ängste vor finanzieller Unsicherheit lassen vermuten, dass vor allem geringere Einkommen ohne Angaben blieben und somit eine Überschätzung der Einkommenssituation nicht ausgeschlossen werden kann.

Weiterhin soll angemerkt werden, dass eine direkte Vergleichbarkeit zwi- 
schen den Ergebnissen des Robert Koch Institutes (RKI) [10] zum subjektiven Gesundheitszustand und den Ergebnissen dieser Arbeit aufgrund leicht unterschiedlicher Erhebungsinstrumenten (5- vs. 6-fach abgestufte Ratingskala) nicht gegeben ist. Vielmehr soll es sich um eine grobe Einordnung der Befunde zur entsprechenden (deutschen) Gesamtbevölkerung handeln. Die Autoren möchten den explorativen Charakter der Studie explizit hervorheben und betonen, dass ihnen bewusst ist, dass aufgrund multipler Testungen ein zufälliges Auftreten einzelner Testergebnisse nicht ausgeschlossen werden kann und herausgearbeitete Hypothesen erneuter Absicherung in Folgearbeiten bedürfen [15]. Im Rahmen dieser Arbeit wurde deshalb auf eine $p$-Wert-Adjustierung verzichtet.

Trotz der beschriebenen Grenzen liefert die vorliegende Arbeit wertvolle Erkenntnisse zur Bewertung der subjektiven Gesundheit, dem Belastungsempfinden und der Zufriedenheit in einer Population, in der Betriebsärzte, Arbeitssicherheit und Abteilungen für Betriebliche Gesundheitsförderung praktisch nicht existent sind.

\section{Ausblick}

Nach subjektiver Einschätzung empfinden TP eine hohe allgemeine Berufszufriedenheit und stehen ihren körperlichen und mentalen Anforderungen sowie ihrem Gesundheitszustand positive gegenüber. Lediglich sozioökonomische sowie psychosoziale Aspekte nehmen eine gesundheitsbelastende Rolle ein. Um aus der subjektiven Belastungssituation Aussagen über die Beanspruchung von TP geben zu können, bedarf es in Folgearbeiten einer Relativierung und Kontextualisierung der subjektiven Einschätzungen anhand objektiver Gesundheitssowie Krankheits- und Verletzungsmerkmale. In diesem Kontext sollten Fragen zu möglichen Healthy-Worker-Effekten berücksichtigt werden. Zusätzlich sollten die Auswirkungen der COVID-19Pandemie auf die Berufssituation untersucht werden, da gravierende Veränderungen sämtlicher Berufsmerkmale im engeren (Unterrichtsdurchführung) so- wie im weiteren Sinne (sozioökonomische Aspekte, Arbeitszufriedenheit etc.) zu erwarten sind.

\section{Fazit für die Praxis}

- Als psychische Stressoren können primär ökonomische und arbeitsorganisatorische Faktoren identifiziert werden.

- Es kann vermutet werden, dass von der eigenen Körperarbeit sowohl eine gesundheitsförderliche als auch -hinderliche Wirkung ausgehen kann. Eine differenziertere Betrachtung der speziellen Körperarbeit im konkreten Arbeitskontext erscheint zwingend erforderlich.

- Eine regelmäßige und bewusste Reflexion berufsbezogener Zufriedenheit und Belastungseinschätzungen könnte eine einfache und ressourcengünstige Methode zur Einschätzung des eigenen subjektiven Gesundheitszustandes darstellen, die eigenständig von den TP im Rahmen eines primärpräventiven Gedankens empfohlen werden kann.

\section{Korrespondenzadresse}

\section{Prof. Dr. Dr. med. Eileen M. Wanke}

Institut für Arbeits-, Sozial- und Umweltmedizin, Goethe-Universität

Theodor-Stern-Kai 7, Haus 9a, 60590 Frankfurt/ Main, Deutschland

wanke@med.uni-frankfurt.de

Funding. Open Access funding enabled and organized by Projekt DEAL.

\section{Einhaltung ethischer Richtlinien}

Interessenkonflikt. M.Schmidt, D. Ohlendorf, R. Reer, D.A. Groneberg und E.M. Wanke geben an, dass kein Interessenkonflikt besteht.

Die Studie befolgt wissenschaftsethische Kriterien. Eine Zustimmung zur ethischen Unbedenklichkeit der Studienprozedur erfolgte seitens der Ethikkommission der Charité - Universitätsmedizin Berlin.

Open Access. Dieser Artikel wird unter der Creative Commons Namensnennung 4.0 International Lizenz veröffentlicht, welche die Nutzung, Vervielfältigung, Bearbeitung, Verbreitung und Wiedergabe in jeglichem Medium und Format erlaubt, sofern Sie den/die ursprünglichen Autor(en) und die Quelle ordnungsgemäß nennen, einen Link zur Creative Commons Lizenz beifügen und angeben, ob Änderungen vorgenommen wurden.
Die in diesem Artikel enthaltenen Bilder und sonstiges Drittmaterial unterliegen ebenfalls der genannten Creative Commons Lizenz, sofern sich aus der Abbildungslegende nichts anderes ergibt. Sofern das betreffende Material nicht unter der genannten Creative Commons Lizenz steht und die betreffende Handlung nicht nach gesetzlichen Vorschriften erlaubt ist, ist für die oben aufgeführten Weiterverwendungen des Materials die Einwilligung des jeweiligen Rechteinhabers einzuholen.

Weitere Details zur Lizenz entnehmen Sie bitte der Lizenzinformation auf http://creativecommons.org/ licenses/by/4.0/deed.de.

\section{Literatur}

1. Antoniou AS, Polychroni F, Vlachakis AN (2006) Gender and age differences in occupational stress and professional burnout between primary and high-school teachers in Greece. Journal of Managerial Psych 21(7):682-690

2. Bogaert I, De Martelaer K, Deforche B, Clarys $P_{\text {, }}$ Zinzen E (2014) Associations between different types of physical activity and teachers' perceived mental, physical, and work-related health. BMC Public Health 14(1):1-9

3. Dahlström M (1997) Physical effort during dance training: a comparison between teachers and students. JDance Med Sci 1(4):143-148

4. Faragher EB, Cass M, Cooper CL (2005) The relationship between job satisfaction and health: a meta-analysis. Occup Environ Med 62(2):105-112

5. IfD Allensbach (2020) Anzahl der Personen in Deutschland, die in der Freizeit tanzen, nach Häufigkeit von 2016 bis 2020 (in Millionen). In Statista. https://de.statista.com/statistik/daten/ studie/171150/umfrage/haeufigkeit-von-tanzenin-der-freizeit/.Zugegriffen: 10. Dez. 2020

6. LiCY, Sung FC (1999) A review of the healthy worker effect in occupational epidemiology. Occup Med 49(4):225-229

7. Michael G, Anastasios S, Helen K, Catherine K, Christine K (2009) Gender differences in experiencing occupational stress: The role of age, education and marital status. Stress Health 25(5):397-404

8. Mölders C, Wanke EM (2019) Wie gesund sind Bewegungen vermittelnde Berufsgruppen? Zentralbl Arbeitsmed 69(4):230-235

9. Nübling M, Stößel U, Hasselhorn HM, Michaelis M, Hofmann F (2006) Measuring psychological stress and strain at work-Evaluation of the COPSOQ Questionnaire in Germany. Psychosoc Med 3:1-14

10. Robert Koch Institut (Hrsg) (2015) Gesundheit in Deutschland. Gesundheitsberichterstattung des Bundes. https://doi.org/10.17886/rkipubl-2015003

11. Robson B, Book A, Wilmerding MV (2002) Psychological stresses experienced by dance teachers. Med Probl Perform Art 17(4):173-177

12. Schmidt M, OhlendorfD, Groneberg DA, Wanke EM (2018) Fit to teach? - Cardiorespiratory capacity, vitamin D3, and Ferritin in physical education teachers with specialization in dance. J Strength Cond Res 00(00):0-0

13. Shivendra D, Kumar MM (2016) A study of job satisfaction and job stress among physical education teachers working in government, semigovernment and private schools. Int J Sports Sci Fit 6(1):89-99

14. SkaalvikEM, Skaalvik S (2009) Does school context matter? Relations with teacher burnout and job satisfaction. Teach Teach Educ 25(3):518-524 
15. Victor A, Elsäßer A, Hommel G, Blettner M (2010) Wie bewertet man die p-Wert-Flut? Dtsch Arztbl Int 107(4):50-56

16. Wanke EM, Clausen K, McCormack M, Groneberg DA (2014) Work-related health problems in dance teachers - a pilot study. Mo J Health Phys Educ Recreat Dance 24:55-66

17. Wanke EM, Quarcoo D, Uibel S, Groneberg DA (2012) Arbeitsunfälle bei Bewegung Vermittelnden, Angestellten Lehrkräften. Evaluation am Beispiel der Tanzpädagogik. Trauma Berufskrankh 14(1):46-50

18. Wanke EM, Schmitter J, Groneberg DA (2012) Analyse und Evaluation der Gesundheitssituation von Bewegung vermittelnden Lehrkräften am Beispiel der Tanzpädagogik. Sportverletz Sportschaden 26(1):49-56

19. Wanke EM, Schmidt M, Oremek G, Bendels M, Ohlendorf D, Groneberg DA (2019) Konsum von Alkohol und Zigaretten bei Tanzpädagogen. Zentralbl Arbeitsmed 69(4):202-209

20. Wanke EM, Schmidt M, Oremek G, Groneberg DA (2020) Work related cardiovascular load in professional dance teachers - a pilot study. J Occup Med Toxicol 15(1):1-10

21. Wanke EM, Schmidt M, Leslie-Spinks J, Fischer A, Groneberg DA (2015) Physical and mental workloads in professional dance teachers. Med Probl Perform Art 30(1):54-60

22. Wu S, Wang R, Zhao Y, Ma X, Wu M, Yan X, He J (2013) The relationship between self-rated health and objective health status: A population-based study. BMC Public Health 13(1):1-9 